\title{
Low- or high-angle Ar ion-beam etching to create ramp-type Josephson junctions
}

\author{
K Verbist $\dagger$, O I Lebedev $\dagger \dagger$, G Van Tendeloo $\dagger$, M A J Verhoeven $\S$, \\ A J H M Rijnders $\S$ and D H A Blank $\S$ \\ $\dagger$ EMAT, University of Antwerp (RUCA), Groenenborgerlaan 171, B-2020 Antwerp, \\ Belgium \\ $\S$ Department of Applied Physics, University of Twente, PO Box 217, 7500 AE \\ Enschede, The Netherlands
}

Received 27 June 1996

\begin{abstract}
The dependence of the ramp geometry on high- or low-angle ion-beam etching, used to structure ramp-type Josephson junctions based on $\mathrm{ReBa}_{2} \mathrm{Cu}_{3} \mathrm{O}_{7-\delta}$ high- $T_{c}$ superconductors, is investigated by cross-section transmission electron microscopy. The surface quality, interfaces and crystal defects are analysed by high-resolution electron microscopy. Technical difficulties to reproducibly obtain the desired slope angle and shape make high-angle ion-beam etching less interesting although the surface quality is comparable and no systematic differences in electrical properties are observed.
\end{abstract}

\section{Introduction}

Josephson junctions (JJs) based on the high- $T_{c}$ superconductor $\mathrm{ReBa}_{2} \mathrm{Cu}_{3} \mathrm{O}_{7-\delta}(\mathrm{ReBCO})$, with $\mathrm{Re}=$ rare earth, can be fabricated in different geometries [1]. The ramp-type geometry applied in a $c$-axis-oriented multilayer of ReBCO favours tunnelling parallel to the $a b$ plane where the coherence length is maximum, thus making it easier to obtain a non-vanishing d.c. supercurrent. Moreover, ramp-type JJs, rather than other junction types, have a geometry (see figure 1) that allows for control of the electrical properties through changes of the barrier layer material and/or thickness. The product of the critical current and the normalstate resistance is an important figure of merit for a JJ. The use of $\mathrm{PrBa}_{2} \mathrm{Cu}_{3-x} \mathrm{Ga}_{x} \mathrm{O}_{7-\delta}(\mathrm{PrBCGaO})$ as barrier layer material results in junctions with a higher normal-state resistance than the use of undoped $\mathrm{PrBa}_{2} \mathrm{Cu}_{3} \mathrm{O}_{7-\delta}(\mathrm{PrBCO})$ as the barrier layer material while it leaves the critical current unaffected [2].

The geometry of ramp-type junctions also facilitates the formation of quantum-interference devices (SQUIDs) with very small loop inductances for digital applications. This is performed by placing two junctions back to back (see figure 2). The loop is then effectively defined by the thickness of the insulating layer and the spacing between the junctions. Both parameters can be well controlled in a standard lithographic process. Thus, sub-picohenry loop-inductances are obtained. This small value enables

$\ddagger$ On leave from: Institute of Crystallography, Leninsky pr. 59, 117333 Moscow, Russia.
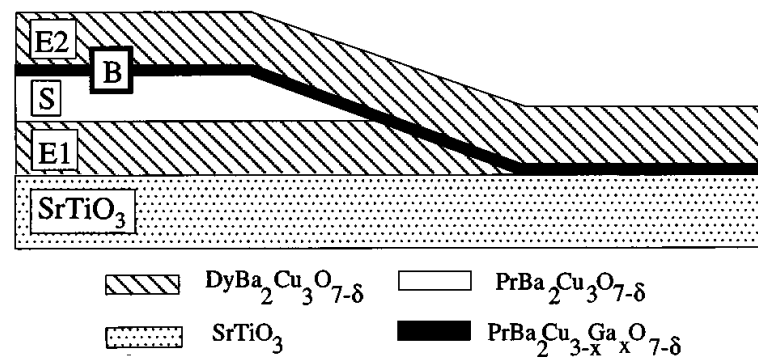

Figure 1. A schematic representation of the ramp-type $\mathrm{JJ}$ geometry with the naming scheme: base electrode (E1), separating layer $(\mathrm{S})$, barrier layer $(\mathrm{B})$ and top electrode (E2).

the formation of a lumped-circuit inductor by structuring a narrow strip-line in the top electrode (in the four-layer part), by which reproducible inductances $L$ are obtained of some $1.4 \mathrm{pH}$. A parallel capacitance $C$, necessary to make the junction hysteretic, is implemented as a wider part in the strip line. Figure 3 shows a micrograph obtained by scanning electron microscopy (SEM) of a realized comparator structure.

This structure has proven to be particularly useful in constructing a periodic-threshold comparator structure for superconducting digital applications [3]. The socalled quasi-one junction SQUID (QOS) is simply a very asymmetric SQUID structure, and behaves in most cases much like a single-junction SQUID. The periodic dependence of the circulating (shielding) current 


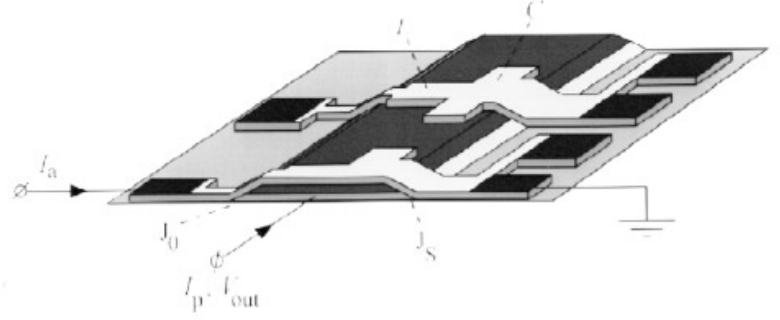

Figure 2. A SQUID structure with a very small loop inductance is created by placing two junctions $J_{0}$ and $J_{s}$ on parallel ramps.

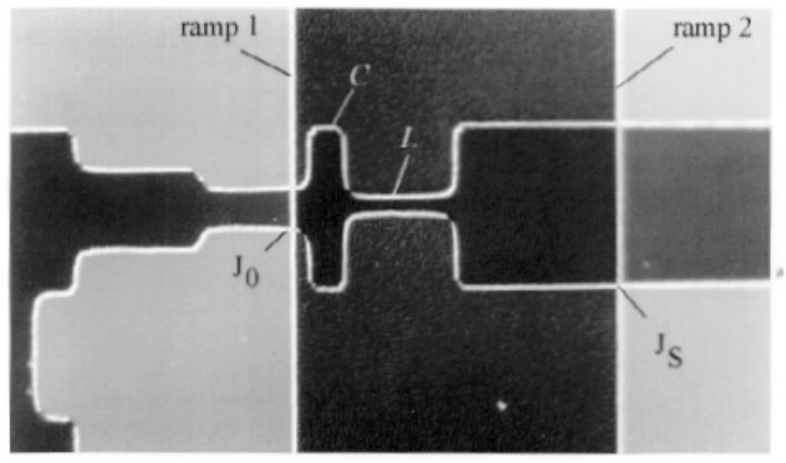

Figure 3. An SEM micrograph (top view) of a realized comparator structure. The two parallel ramp edges are clearly visible as well as the implementation of the loop inductance $L$ and parallel capacitor $C$.

in a superconducting loop, as a consequence of flux quantization, is useful for digital applications. The way to define a digital ' 1 ' or ' 0 ' (figure 2) is then by sensing the direction of the circulating current in the digitizing junction $J_{0}$ which in turn is a periodic function of the analogue input $I_{a}$. This is done by adding a very high and sharp current pulse $\left(I_{p}\right)$ to the circuit that is just able to switch the large sampling junction $\left(J_{s}\right)$ in the asymmetric SQUID to the voltage state, if the shielding current is clockwise. Thus, a voltage pulse $\left(V_{\text {out }}\right)$ appears (' 1 ') and in the cases where the shielding current is counter clockwise, the circuit does not come into the voltage state (' 0 ').

In a binary code, each bit is also a periodic function of the input signal, with a period doubling for each higher significant bit. To construct an $n$-bit Gray-code analogueto-digital converter (ADC) one just has to divide the input signal (by e.g. an $\mathrm{R}-2 \mathrm{R}$ resistor ladder) into $n$ identical QOS comparators. The advantage of superconducting ADCs is the promise of much higher input bandwidths (up to $\mathrm{THz}$ frequencies). For 'flash'-ADCs, by virtue of the intrinsic periodicity of the supercurrent, there is an additional advantage that only $n$ comparators are needed, instead of the $2^{n}-1$ needed in a semiconductor architecture.

JJs with a ramp-type geometry are prepared by structuring a ramp edge in a ReBCO base electrode and separating layer. Afterwards a barrier layer and a top electrode are epitaxially grown over this structured ramp. Different techniques such as wet chemical etching [4], shadow mask technique [5] and ion-beam etching [6] are known to structure ramp edges in ReBCO layers. Using ion-beam etching the process of ramp-structuring is more controllable. When ramp structures are made by ion-beam etching usually a photoresist mask is used to protect the parts which do not have to be ion-beam etched. Etching of a strip of photoresist with the ion beam perpendicular to the film surface yields, on the two sides of the strip, ramp edges produced under identical conditions of ionbeam incidence. Etching with an incident ion beam not perpendicular to the original surface allows the tuning of the slope angle. However, one line of photoresist then gives rise to two ramp edges fabricated under different conditions of ion-beam incidence to the final ramp surface. The ramp which is facing away from the ion-beam gun is created under low-angle ion-beam incidence and the ramp which is facing towards the ion-beam gun is created under high-angle ion-beam incidence. The etching rate and the damage caused by ion-beam etching depend on the angle of incidence of the ion-beam [7]. The surface quality and morphology of the ramp edge and the subsequent growth of the barrier layer strongly influence the electrical properties of the JJ. Therefore the question was put forward of whether the surface quality and morphology of the ramp edges created using low- and high-angle ion-beam etching differ and whether both types are usable to make reproducible JJs with the desired electrical properties. This question is especially important for the realization of a comparator structure consisting of two back-to-back JJs.

The amount of work published on the microstructural characterization of ramp-type JJs with PrBCO or PrBCGO as barrier layer is limited. Lebedev et al [8] report an increasing slope angle with increasing distance from the substrate, presumably caused by the different etching rates of YBCO and PrBCO. The ramp structures they report on were created with perpendicular ion-beam etching. Wen et al [9] report on the microstructure of ramp-type JJs for different substrates. The film deposition and ionbeam etching procedures are largely identical to the one reported here. The etching angle used to produce the DyBCO/PrBCO/DyBCO ramps was $45^{\circ} \dagger[10]$. They mainly observed that ramps facing away from the ion gun have a dimple in the substrate near the base of the junction and that less substrate material is removed for the ramps facing towards the ion gun than on the neighbouring ramps. This was explained by the increased flux of ions at the foot of the junction due to reflection. The barrier layer of ramps facing away from the ion source has in general less sharp interfaces but the eventual negative influence on the properties of the $\mathrm{JJ}$ is not clear.

We have studied the geometry, surface roughness and surface quality of ramp-type edges of high- $T_{c}$ superconducting $\mathrm{JJ}_{\mathrm{s}}$ with a PrBCGO barrier layer to reveal the differences between edges created with lowor high-angle ion-beam etching by means of electrical measurements and cross-section transmission electron microscopy (TEM). The consequences of high- and lowangle ion-beam etching on the continuous overgrowth of the barrier and top electrode layer are discussed.

$\dagger$ The slope of ramps, defined by ion etching of photoresist masks, differs from the angle of incidence of the ion beam, due to the difference in etch rate. An angle of incidence of $45^{\circ}$ results in a slope with an angle of $20^{\circ}$. 


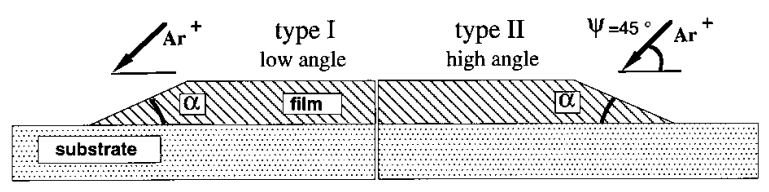

Figure 4. The geometry used to create the ramp structures by ion-beam etching of one photoresist line resulting in two kinds of edges: defined here as type I and type II.

\section{Experimental methods}

All layers have been deposited by off-axis radiofrequency magnetron sputtering on $\mathrm{SrTiO}_{3}$ substrates from single stoichiometric targets. The geometry of the ramp-type junctions is shown schematically in figure 1 as well as the naming scheme used in this text. The base electrode of $\mathrm{DyBa}_{2} \mathrm{Cu}_{3} \mathrm{O}_{7-\delta}$ (E1) and the separating layer of $\mathrm{PrBa}_{2} \mathrm{Cu}_{3} \mathrm{O}_{7-\delta}$ (S) have been deposited in one vacuum cycle. The surface was covered by a $1.2 \mu \mathrm{m}$ thick photoresist mask of parallel lines, aligned along one of the cube axes of the $\mathrm{SrTiO}_{3}$ substrate. The ramp-type structures were created using an argon Kaufmann source ( $V=500 \mathrm{eV}, I=10 \mathrm{~mA}, 3 \mathrm{~cm}$ diameter) under an angle of $\psi=45^{\circ}$. After chemical removal of the photoresist, the surface of etched and unetched parts was cleaned by removing 20 to $30 \mathrm{~nm}$ of material with the same source operating $1 \mathrm{~min}$ at $500 \mathrm{eV}$ and $3 \mathrm{~min}$ at $50 \mathrm{eV}$. Without breaking the vacuum the sample was transported from the ion-etch chamber into the sputtering chamber. Inside the latter it was heated in the sputter atmosphere $(20 \mathrm{~Pa}$ Ar: $\mathrm{O}_{2}=1: 1,0.1 \% \mathrm{H}_{2} \mathrm{O}$ gas) at $790{ }^{\circ} \mathrm{C}$ for $1 \mathrm{~h}$ to achieve re-oxygenation of the surfaces. Subsequently the barrier layer of $\mathrm{PrBa}_{2} \mathrm{Cu}_{3-x} \mathrm{Ga}_{x} \mathrm{O}_{7-\delta}$ (B) and the top electrode of $\mathrm{DyBa}_{2} \mathrm{Cu}_{3} \mathrm{O}_{7-\delta}$ (E2) were deposited. Afterwards a $\mathrm{SrTiO}_{3}$ cover layer was deposited (ex situ) with laser ablation. This layer has no technological purposes and served only as a marker of the final growth surface for the electron microscopy (EM) study. The samples studied here are made on purpose with a multiple-mask (spacing $10 \mu \mathrm{m}$ masked, $10 \mu \mathrm{m}$ unmasked) using the same deposition procedures as the operational junctions, so that cross-section EM samples contain many junctions. Also ramp structures, created using the same conditions but without the barrier and top electrode layer were investigated. We will refer to the latter as bare ramps. The critical current densities were determined on ramp contacts: these are ramp-type junctions without any barrier layer. More details on the fabrication process have been reported elsewhere [2,11].

The angle between the ion beam and the original film surface will be defined as $\psi$ and the angle between the ramp surface and the interface with the substrate as $\alpha$ (figure 4). So ramps facing away from the ion source (defined here as type I ramps), with e.g. a slope angle $\alpha=15^{\circ}$, are created with an incident ion beam of $30^{\circ}$ with respect to the final ramp surface. Ramps facing towards the ion source (defined here as type II), with the same slope angle $\alpha=15^{\circ}$, are etched with the incident ion beam at $120^{\circ}$ to the final ramp surface.

There is an inhomogeneous distribution of the ion-beam flux resulting in a difference of the amount of material

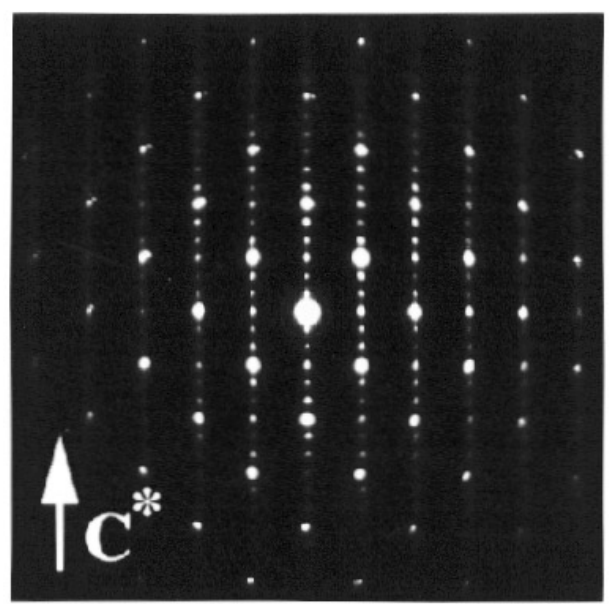

Figure 5. The [100] diffraction pattern taken from the ramp area showing the $c$-axis orientation of all ReBCO layers and the epitaxial relation with the $\mathrm{SrTiO}_{3}$ substrate.

removed over the $1 \times 1 \mathrm{~cm}$ substrate. It can happen that $20 \mathrm{~nm}$ of $\mathrm{SrTiO}_{3}$ is etched away at the foot of a certain edge while $20 \mathrm{~nm}$ of the base electrode still remains near the foot of another edge, spaced by nearly $1.4 \mathrm{~cm}$ on the substrate from the first edge. As a result the etching procedure can be incomplete, i.e. a part of DyBCO not covered by photoresist remains on the substrate. However, this problem is specific to the multiple mask used for this EM study with 1000 edges per centimetre since for electrical measurements only five junctions are created on a substrate. Adjacent edges have always been investigated to avoid the influence of the inhomogeneous distribution of the ion-beam flux.

Cross-section samples for EM are mechanically polished with a tripod to a thickness of $15 \mu \mathrm{m}$ on diamond lapping foils. Further thinning is done in a Balzers RES 010 using Ar ion milling of $4.5 \mathrm{keV}$ and $0.5 \mathrm{~mA}$ at an angle of $15^{\circ}$. Once colour fringes start to appear the conditions are changed to $3 \mathrm{keV}, 0.3 \mathrm{~mA}$ and $10^{\circ}$. High-resolution electron microscopy (HREM) and electron diffraction (ED) investigations are performed on a JEOL 4000 EX and a Philips CM 20 microscope. All crosssection EM micrographs shown here are recorded with the [001] axis of the ReBCO layers perpendicular to the electron beam.

\section{Results}

The investigated ramps are generally of good quality with the expected geometry. All layers are $c$-axis oriented and the overgrown barrier layers and top electrodes have epitaxial $c$-axis orientation for all the analysed slope angles. This is shown in a representative diffraction pattern taken over the whole ramp area (figure 5). The different layers can clearly be distinguished in low magnification images such as figure 6 . The interfaces between the various layers are sharp and flat. The high quality is also reflected in the electrical properties [2] and indicates that the fabrication procedure is close to being optimized.

A ramp can be divided into three zones with different slope angles: a foot (f) near or in the substrate, a middle part 

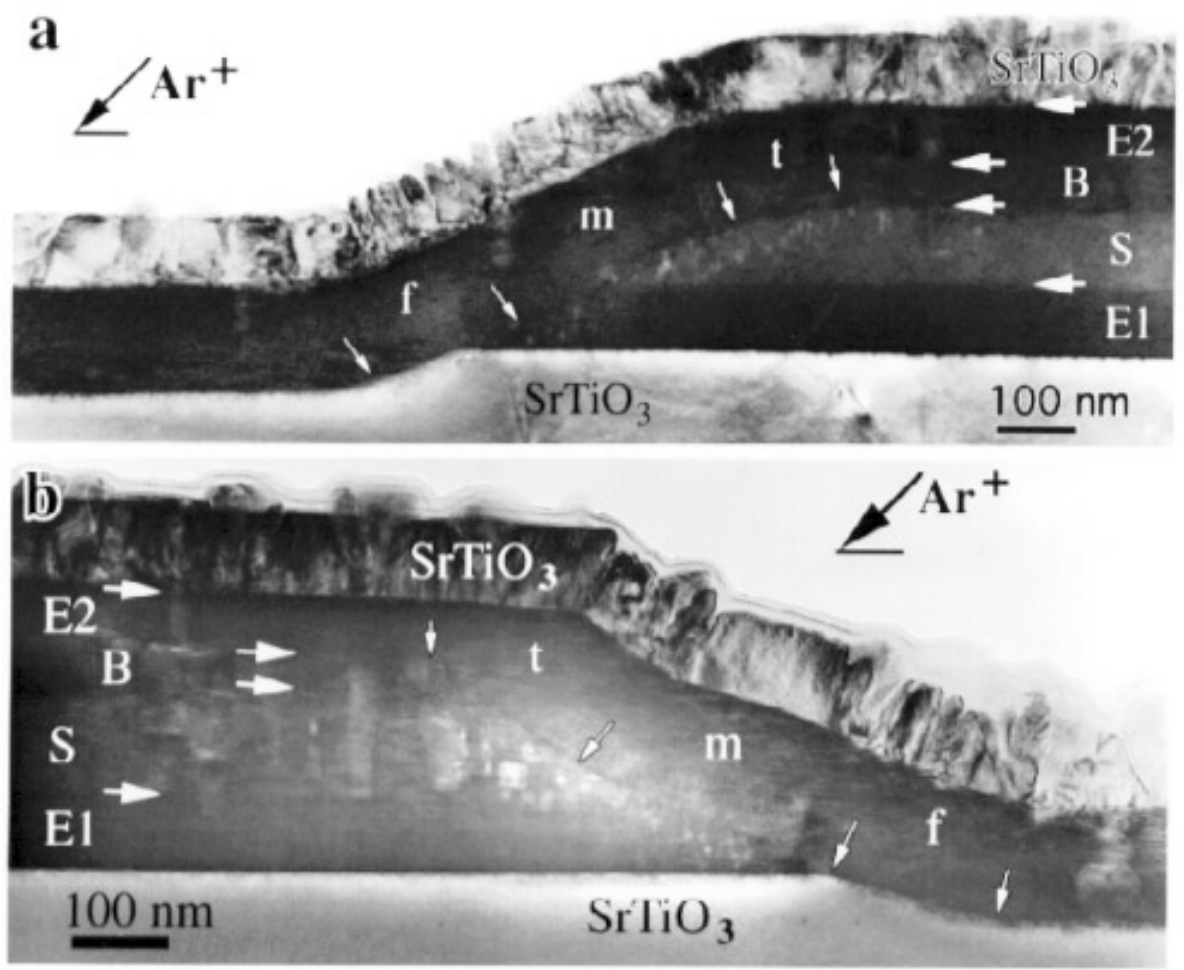

Figure 6. Ramp-type JJs created using low-angle ion-beam etching (a) and high-angle ion-beam etching (b) of the same photoresist line. The three zones, top $(\mathrm{t})$, middle $(\mathrm{m})$, foot $(\mathrm{f})$ are indicated. Horizontal arrows indicate the interfaces between various layers. The small arrows point to the etched film surface. There is a difference in the roughness of the overgrown layers (B and E2) between types I and II.

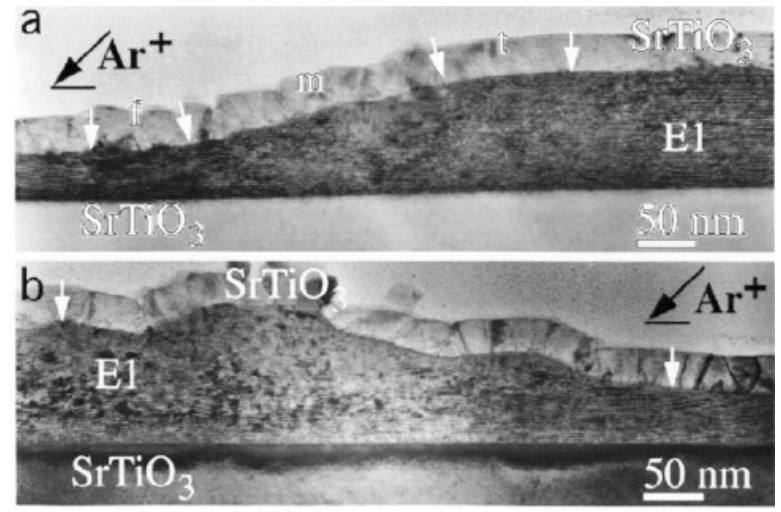

Figure 7. Bare ramps created using low-angle (a) and high-angle (b) ion-beam etching where the difference between the two types is obvious. No dip at the foot of the edge can be observed.

(m) with the interfaces between barrier layer and electrodes which is important for the actual tunnelling and a top (t) where the edge joins the unetched film part. These three zones are indicated on the low-magnification image of a representative example of a type I ramp edge in figure 6. Although these are complete ramp-type JJs (with B and E2 layers), still the shape of the ramp edge, created by ion etching in the bottom layers $\left(\mathrm{SrTiO}_{3}, \mathrm{E} 1\right.$ and $\left.\mathrm{S}\right)$ and indicated by small arrows in figure 6 , can be analysed. This ramp edge, etched facing away from the ion source (figure 6(a)), has a linear slope with an angle $\alpha=28^{\circ}$ in the

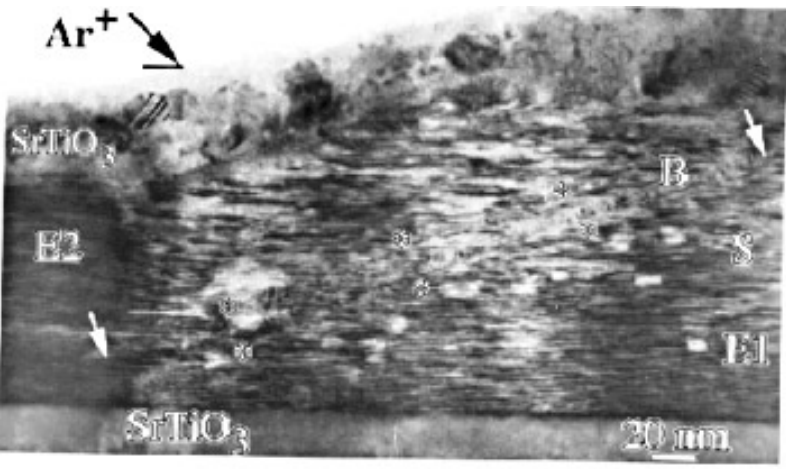

Figure 8. A ramp-type $\mathrm{JJ}$ created with high-angle ion-beam etching. The slope is linear with $\alpha=21^{\circ}$ and the overgrown layers are continuous and have a constant thickness. The barrier layer interfaces are marked by asterisks and the slope by arrows.

middle part, a slightly smaller slope angle $\left(22^{\circ}\right)$ at the foot of the edge in the substrate and a rounded top with a slope angle $\alpha=15^{\circ}$. The B and E2 layers grow over the edge in a continuous smooth way and the final growth surface of the E2 layer follows the shape of the etched ramp.

Figure 6(b) shows the type II ramp edge adjacent to the type I ramp edge shown in figure 6(a). The three zones with the respective slope angles can be described as follow: the top at the interface with the separating layer is concave with an average angle of $35^{\circ}$, the middle part (situated near the interface $\mathrm{S} / \mathrm{E} 1$ ) is linear with a slope of $20^{\circ}$ and the 


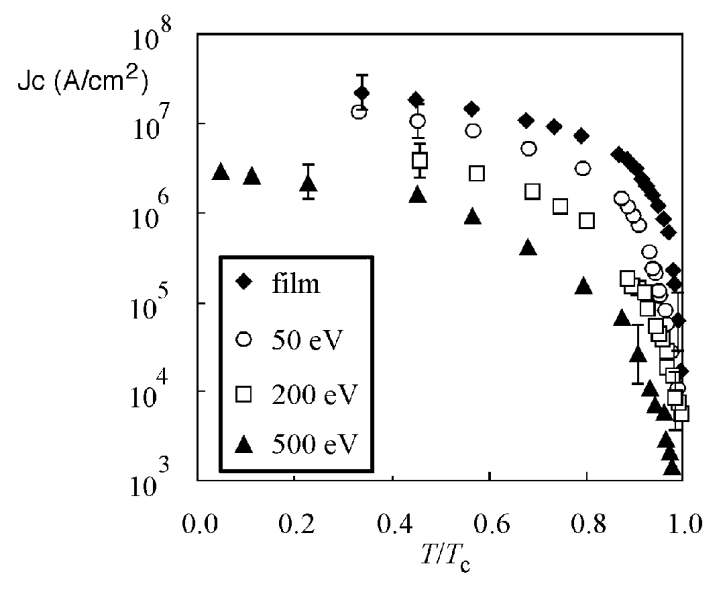

Figure 9. $J_{C}\left(T / T_{c}\right)$ of DyBCO/DyBCO ramp contacts of type I $\left(T_{c}=89 \mathrm{~K}\right)$, cleaned with different Ar-ion kinetic energies to be compared with pristine DyBCO films.

foot in the substrate has an angle of $15^{\circ}$. Typically the top of the edge is not rounded for type II ramp edges but is angular. The final growth surface of E2 is rough and consists of horizontal plateaus and steep edges with angles of $30^{\circ}$ to $35^{\circ}$.

For low slope angles the difference between type I and type II is already apparent on bare ramps without overgrown layers, as can be seen in figure 7. The top and bottom of the slopes are marked by white arrows. The type I edge (a) is smooth with a linear slope of $11^{\circ}$ while the type II edge (b) is very rough $\left(\langle\alpha\rangle^{2} \leq 10^{\circ}\right)$. Usually the unevenness on the surface of the type II ramps which can be up to $10 \mathrm{~nm}$, occurs near the top with the unetched part and near the foot of the edge. Notice there is no dip at the foot of the edge in both cases. There are situations in which ionbeam etching using a high angle results in a linear slope, indicated by arrows in figure 8 , and a smooth surface on which a continuous smooth overgrowth can take place.

The interfaces between the electrode layers $\mathrm{E}$ and the barrier layer B are the heart of the JJs and determine, together with possible defects in the B layer, the electrical characteristics of the junction. The possible damage at the ramp interface induced by the Ar ion-beam etching and cleaning procedure is an important factor in determining the interface quality. This damage was found to be very dependent on the acceleration voltage (figure 9). Ramp contacts showed clear weak-link behaviour when cleaned with ions having kinetic energies of $500 \mathrm{eV}$ and $200 \mathrm{eV}$ and large periodic modulation (up to 50\%) of the critical current on weak magnetic fields. The $500 \mathrm{eV}$ cleaned ramp contacts showed a reduction of one order of magnitude in $J_{c}$ with respect to the thin-film value. When they had been cleaned subsequently for some time at $50 \mathrm{eV}$, this weaklink behaviour was not observed and the critical current density was found to be very close to the ideal value for DyBCO thin films.

The interfaces E1/B and B/E2, as well as the quality of all layers, were investigated using HREM. More structural and crystallographic details are reported in $[12,13]$. No amorphous layer is present at the interface between $\mathrm{E}$ and $\mathrm{B}$ layers and no secondary phases are detected. Usually the interface E1/B is more easily visible on the low magnification and HREM micrographs than the interface $\mathrm{B} / \mathrm{E} 2$ due to the inequivalent growth conditions. The $\mathrm{E} 1 / \mathrm{B}$ interface contains more defects such as $\mathrm{Cu}-\mathrm{O}$ double layers and anti-phase boundaries (APBs) due to the ex situ processing. A typical HREM image of the interface of the B layer with the E1 layer, for a type I ramp, is shown in figure 10. The HREM contrast difference between DyBCO and PrBCGO is very small. The interface can be recognized more easily because APBs, originating at the substrate, end at the interface with the B layer. The E1/B interfaces of the type II ramps show no structural differences in defect density as compared to type I ramps. We observed no significant difference in critical current densities between ramp contacts that were facing (type II) and facing away (type I) from the ion beam during etching respectively (figure 11).

\section{Discussion}

The ramp edges created with low- and high-angle ionbeam etching are clearly different in shape and slope angle. For neighbouring edges the slope angle of the middle part is always higher for type I edges. The morphological differences are summarized in table 1 . Systematic differences in amount of substrate material removed at the foot of the junction, as reported by Wen et al [9], were not detected. However, it is important to etch far enough so as to remove all E1 material, which is not protected by photoresist, because otherwise a contact E1/B/E2 exists along the $c$ axis of the film as shown in figure 7. This could give rise to tunnelling along the $c$ axis and should therefore be avoided.

The angle $\psi$ between the ion beam and the original surface, which was maintained at $45^{\circ}$ in this study, yields ramps with slope angles $\alpha$ varying from $10^{\circ}$ to $35^{\circ}$ depending on the etch time and the position on the substrate. The fact that the ramps have a slope angle different from $45^{\circ}$, especially for the ramps facing away from the ion source, can be understood if the etch rate and the shadow of the photoresist are taken into account. The etch rates for the reported etch conditions are: $60 \mathrm{~nm} \mathrm{m^{-1 }}$ for photoresist, $30 \mathrm{~nm} \mathrm{~min}{ }^{-1}$ for $\mathrm{ReBCO}$ and $15 \mathrm{~nm} \mathrm{~min}^{-1}$ for $\mathrm{SrTiO}_{3}$. The thickness of the photoresist layer $(1.2 \mu \mathrm{m})$ is much higher than the film thickness $(100-150 \mathrm{~nm})$ so throughout the etching process a shadow effect will be important for the edges facing away from the ion source. The rounded top part of type I edges is a consequence of the shadow effect. This effect is absent for high-angle edges and as a result the top part of these edges is always sharp and like a corner.

The spectacular difference between edges of type I and II with low slope angles of $10^{\circ}$ to $12^{\circ}$ is also related to the shadow effect. The side surface of the photoresist line is not necessarily smooth. A roughness on this surface can reflect the ions and result in a locally higher flux which created the unevenness in the type II ramps. For type I ramps, the possible roughness lies in the shadow and plays no role. 


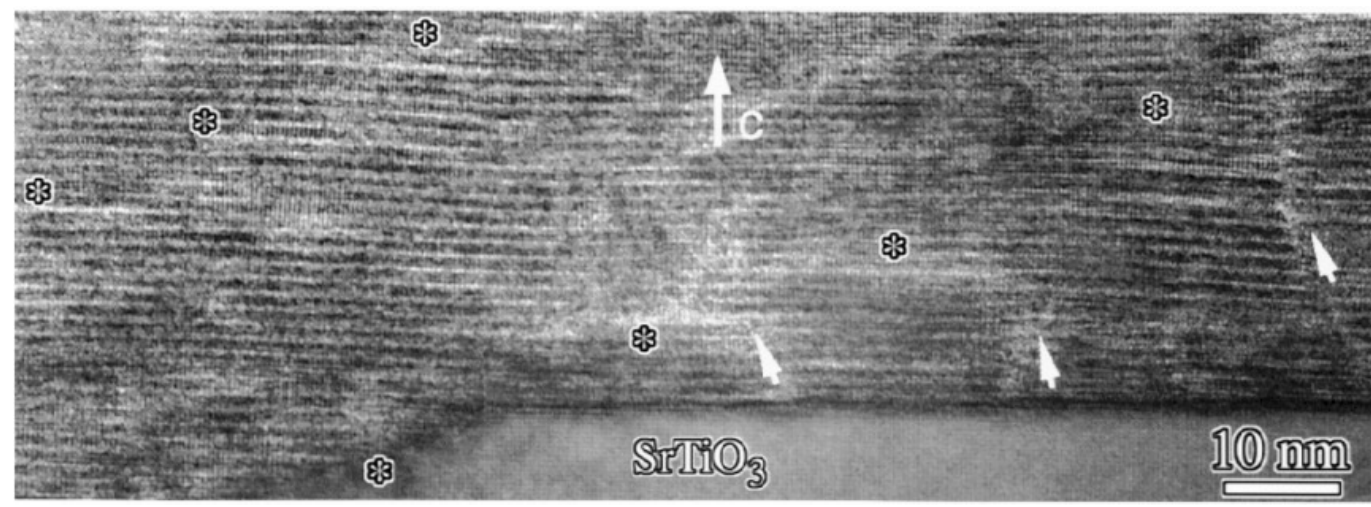

Figure 10. HREM of the barrier-electrode interfaces, marked by asterisks, for a type I ramp. Anti-phase boundaries in the base electrode, which end at the interface with the barrier layer, are indicated by arrows.

Table 1. The morphology of type I and II ramp edges.

\begin{tabular}{|c|c|c|}
\hline & Type I: low angle & Type II: high angle \\
\hline $\begin{array}{l}\text { Top part } \\
\text { Middle part } \\
\text { Foot }\end{array}$ & $\begin{array}{l}\text { Rounded } \\
\text { Always linear } \\
\text { < slope angle in substrate } \\
\text { stepped foot in substrate }\end{array}$ & $\begin{array}{l}\text { Sharp corner } \\
\text { Bumpy, linear or concave } \\
<\text { slope angle in substrate } \\
\text { no dip }\end{array}$ \\
\hline
\end{tabular}

As a consequence the restrictions on the photoresist line quality are more severe for type II ramps.

The investigation of different samples with the special geometry allows a statistical approach to determine average ramp profiles for different slope angles. These ramp profiles, which represent the ramp-edge geometry with different amounts of material removed, are in fact snapshots of the evolution of the ramp-edge geometry as a function of etch time. The evolution of the ramp profile for lowangle ion-beam etching is shown in figure 12(a). The arrowheads mark the unetched surface which was covered by the photoresist layer. For all analysed slope angles, between $11^{\circ}$ and $28^{\circ}$, a linear slope is obtained. The slope angle of the foot is lower when it extends in the substrate due to the lower etch rate of $\mathrm{SrTiO}_{3}$. On this type I ramp a smooth continuous overgrowth is always achieved. This means that for low-angle ion-beam etching, the final slope angle, obtained by etching an aligned photoresist line on the substrate for a certain time, is not important to ensure a smooth continuous B layer.

The evolution of the ramp profile for high-angle ionbeam etching is shown in figure 12(b). In the beginning the ramp surface is very rough then it evolves to a smooth surface with a linear slope. If the etching procedure is continued, a concave edge with an extended foot is created. A smooth continuous overgrowth is only observed on the linear ramp edge with an angle of $21^{\circ}$ as shown in figure 8 .

These results indicate that for high-angle ion-beam etching the etch time is critical and also the position and quality of the photoresist line on the substrate are important. The technical difficulties in obtaining reproducibly linear slopes with high-angle ion-beam etching render its application in the fabrication of ramp-type JJs less interesting.

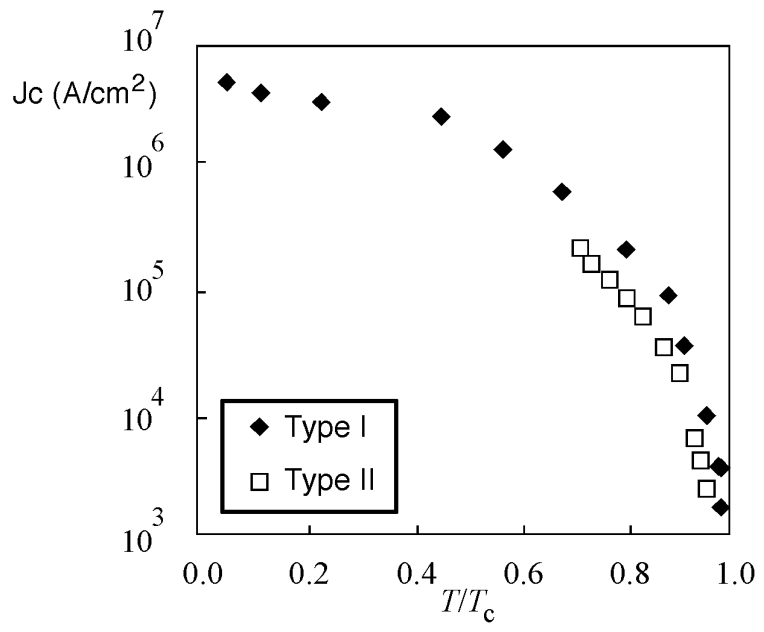

Figure 11. $J_{c}\left(T / T_{c}\right)$ of DyBCO/DyBCO ramp contacts of type I and II cleaned with $500 \mathrm{eV}$ Ar ions.

Moreover, a constant B layer thickness is desired to minimize the spread on the electrical properties. We observed that concave edges always give rise to a rough overgrowth of the B layer with a large spread in thickness measured in the $a b$ plane. Some straightforward considerations can explain the rough covering on concave ramp edges. When looking at a schematic representation of edges on a unit cell scale, it is obvious that a concave edge contains long vertical steps with only small horizontal plateaus. The nucleation sites of the B layer are located in the shallow ridges between steps and plateaus as was previously reported [14]. This knowledge combined with the growth speed anisotropy of ReBCO shows that a smooth covering is more difficult to achieve on concave edges. 


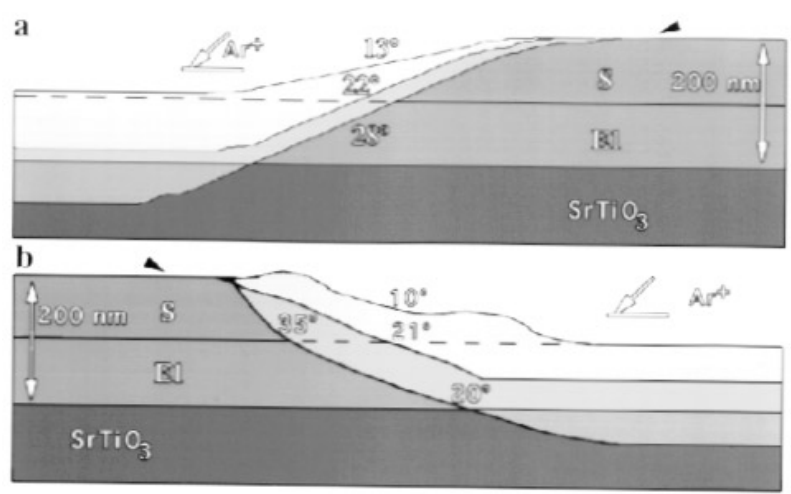

Figure 12. Evolution of the ramp profile as a function of the etch time for low-angle ion-beam etching (a) and for high-angle ion-beam etching (b). The arrowheads mark the pristine surface of the ReBCO film which is rounded for type I (a) and angular for type II (b). The middle part of the type I slope is linearly independent of the etch time (a). An etching optimum with a linear slope exists for type II ramps (b); however, if the etching is continued a concave edge with an extended foot is created.

The restricted size of the plateaus prohibits the growth in the plane of the plateau while nucleation sites on different plateaus have not yet coalesced because, taking into account the lower growth rate in the $c$ direction, they cannot overcome the high steps in the $c$ direction. If plateau $\mathrm{A}$, below a certain plateau $\mathrm{B}$, is fully covered and the domain height on plateau A is sufficient, only then can the domain on plateau B grow further in the plane of the plateau. This effect can be responsible for the rough overgrowth of the B and top layer on concave type II edges and renders concave edges less suited to obtain reproducible JJs.

\section{Conclusion}

Our study shows that ramp-type JJs can be produced by low- or high-angle ion-beam etching. The interface quality is comparable for both cases and no systematic differences in the electrical properties could be found. Therefore the comparator structure can be produced by etching in one step a single photoresist line using for one JJ high-angle etching and for the other JJ low-angle etching. However, one should realize that the technical difficulties to obtain, in a reproducible way, the desired slope shape and slope angle are greater for high-angle ion-beam etching.

\section{Acknowledgments}

The authors would like to thank L Rossou and G Stoffelen for the TEM specimen preparation. This research is part of the EC project on Novel Superconductors No. CHRX-CT94-0461. This text presents research results of the Belgian Inter University Attraction Poles programme initiated by the Belgian State, Prime Minister's Office of Science Policy Programming. O I Lebedev acknowledges the fellowship obtained from the latter. Scientific responsibility is assumed by the authors.

\section{References}

[1] Kuprianov M Yu and Likharev K K 1990 Sov. Phys. Usp. 33340

[2] Verhoeven M A J, Golubov A A, Gerritsma G J and Rogalla H 1995 IEEE Trans. Appl. Supercond. 52095

[3] Wiegerink R J, Verhoeven M A J, Gerritsma G J and Rogalla H 1995 IEEE Trans. Appl. Supercond. 53452

[4] Faley M I, Poppe U, Soltner H, Jia C L, Siegel M and Urban K 1993 Appl. Phys. Lett. 6315

[5] Bercherer Th, C. Stölzel, Adrian G and Adrian H 1993 Phys. Rev. B 4714650

[6] Gao J, Aarnink W A M, Gerritsma G J and Rogalla H 1990 Physica C 171126

[7] Barna A 1992 Mater. Res. Soc. Symp. Proc. (Pittsburgh: Materials Research Society) vol 245 p 3

[8] Lebedev O I, Vasiliev A L, Kiselev N A, Mazo L A, Gaponov S V, Paveliev D G and Strikosky M D 1992 Physica C 198278

[9] Wen J G, Koshizuka N, Traeholt C, Zandbergen H W, Reuvekamp E M C M and Rogalla H 1995 Physica C 255293

[10] Blank D H A, Rijnders A J H M, Verhoeven M A J, Bergs R M H, Rogalla H, Verbist K, Lebedev O I, van Tendeloo G 1996 J. Alloys Compounds at press

[11] Verhoeven M A J, Gerritsma G J and Rogalla H 1995 Proc. Eucas 1995

[12] Verbist K, Lebedev O I, Van Tendeloo G, Verhoeven M A J, Rijnders A J H M and Blank D H A Proc. EUREM 1996 (Dublin)

[13] Verbist K, Lebedev O I, Van Tendeloo G, Verhoeven M A J, Rijnders A J H M and Blank D H A unpublished

[14] Verhoeven M A J, Moerman R, Bijlsma M E, Rijnders A J H M, Blank D H A, Gerritsma G J and Rogalla H 1996 Appl. Phys. Lett. 681276 